\title{
HPLC Analysis of Chiral Compounds
}
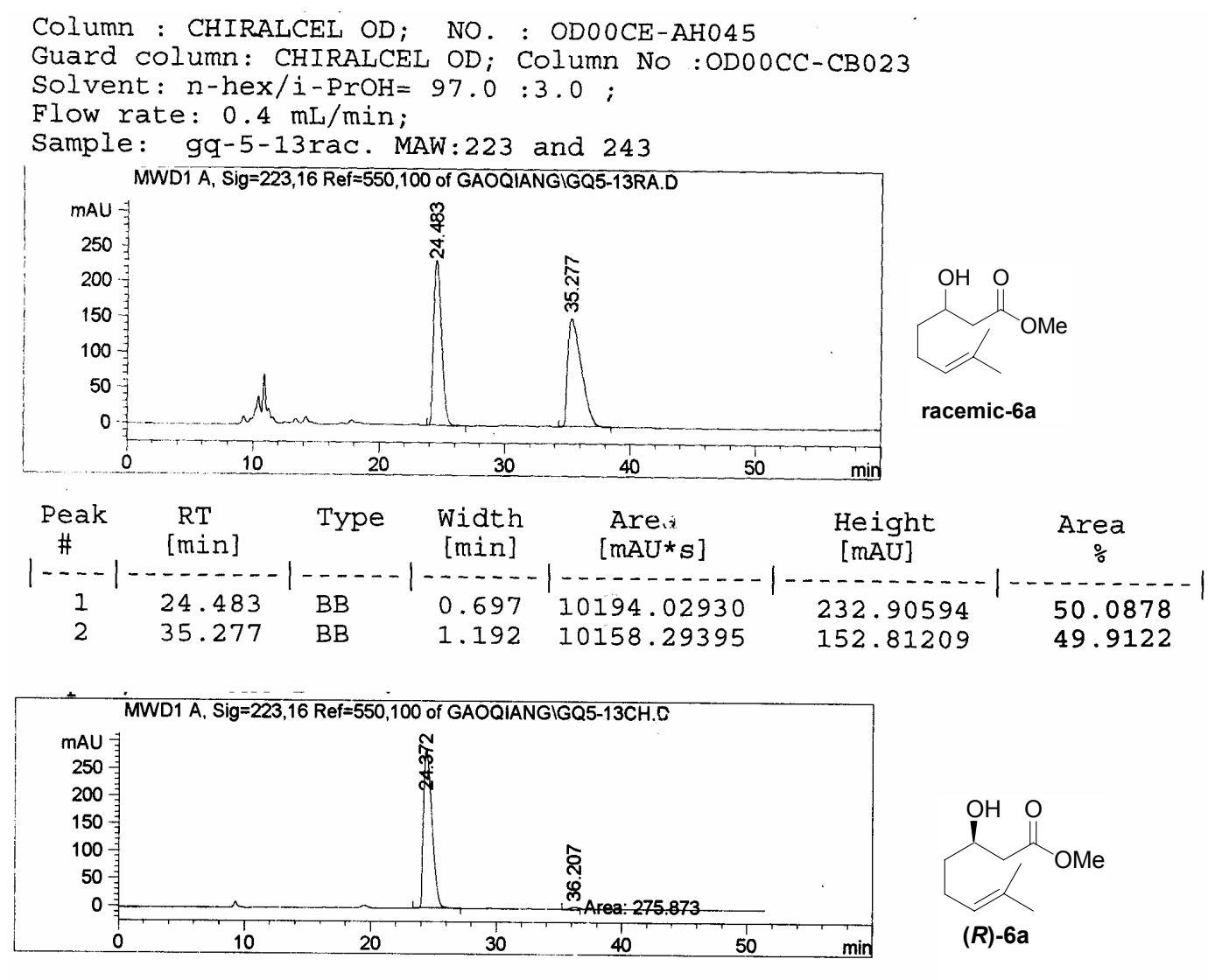

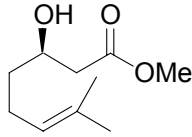

$(R)-6 \mathbf{a}$

\begin{tabular}{|c|c|c|c|c|c|c|}
\hline $\begin{array}{c}\text { Peak } \\
\#\end{array}$ & $\begin{array}{l}\mathrm{RT} \\
{[\mathrm{min}]}\end{array}$ & Type & $\begin{array}{l}\text { Width } \\
\text { [min] }\end{array}$ & $\begin{array}{l}\text { Area } \\
{\left[\mathrm{mAU}{ }^{*} \mathrm{~s}\right]}\end{array}$ & $\begin{array}{l}\text { Height } \\
\text { [mAU] }\end{array}$ & $\begin{array}{c}\text { Area } \\
\frac{\circ}{0}\end{array}$ \\
\hline $\begin{array}{l}1 \\
2\end{array}$ & $\begin{array}{l}24.372 \\
36.207\end{array}$ & $\begin{array}{l}\text { BB } \\
\text { MM }\end{array}$ & $\begin{array}{l}0.755 \\
0.925\end{array}$ & $\begin{array}{r}13573.48145 \\
275.87308\end{array}$ & 287.49783 & 98.0080 \\
\hline
\end{tabular}


Column : ODOOCE-AH045;

Guard column: CHIRALCEL OD; Column No :OD00CC-CB023

Solvent: $\mathrm{n}$-hex/i-PrOH $=90: 10.1 \mathrm{ml} / \mathrm{min}$

Sample: gq-4-177p2 racemic

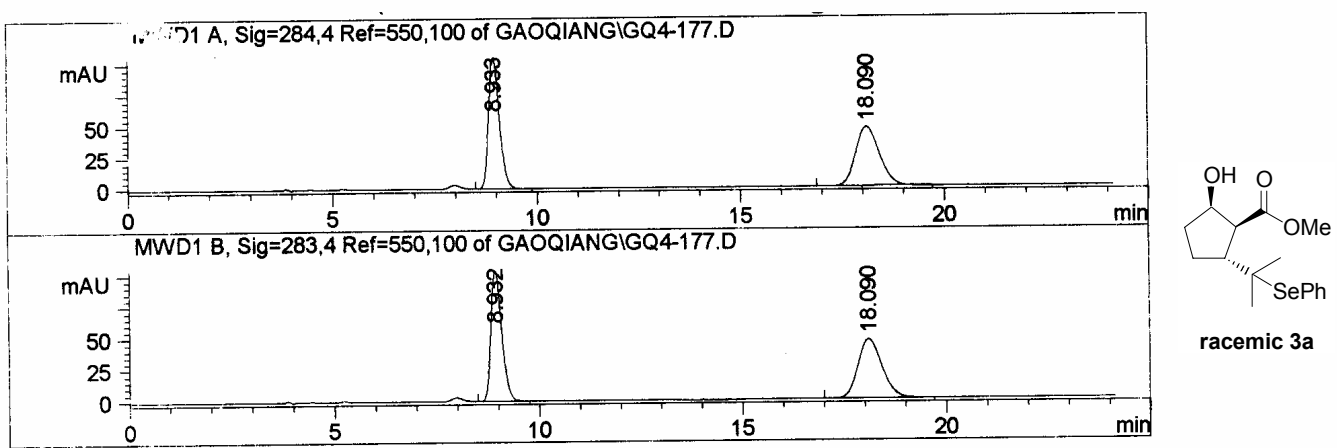

Signal 1: MWD1 A, Sig=284, 4 Ref $=550,100$

\begin{tabular}{|c|c|c|c|c|c|c|}
\hline $\begin{array}{l}\text { Peak } \\
\text { \# }\end{array}$ & $\begin{array}{l}\mathrm{RT} \\
{[\mathrm{min}]}\end{array}$ & Type & $\begin{array}{l}\text { Width } \\
\text { [min] }\end{array}$ & $\begin{array}{l}\text { Area } \\
{\left[\mathrm{mAU}{ }^{*} \mathrm{~s}\right]}\end{array}$ & $\begin{array}{l}\text { Height } \\
\text { [mAU] }\end{array}$ & $\begin{array}{c}\text { Area } \\
\frac{\circ}{0}\end{array}$ \\
\hline & $\begin{array}{r}8.933 \\
18.090\end{array}$ & $\begin{array}{l}\text { VV } \\
\text { BV }\end{array}$ & $\begin{array}{l}0.287 \\
0.638\end{array}$ & $\begin{array}{l}1960.58862 \\
1956.21960\end{array}$ & $\begin{array}{r}105.75696 \\
47.65023\end{array}$ & $\begin{array}{l}50.0558 \\
49.9442\end{array}$ \\
\hline
\end{tabular}

Signal 2: MWD1 B, Sig=283,4 Ref $=550,100$

\begin{tabular}{|c|c|c|c|c|c|c|}
\hline $\begin{array}{l}\text { Peak } \\
\#\end{array}$ & $\begin{array}{l}\mathrm{RT} \\
\text { [min] }\end{array}$ & Type & $\begin{array}{l}\text { Width } \\
\text { [min] }\end{array}$ & $\begin{array}{c}\text { Area } \\
{\left[\mathrm{mAU}{ }^{*} \mathrm{~s}\right]}\end{array}$ & $\begin{array}{l}\text { Height } \\
\text { [mAU] }\end{array}$ & $\begin{array}{c}\text { Area } \\
\%\end{array}$ \\
\hline $1-$ & 8.932 & $\mathrm{~PB}$ & 0.287 & 1914.88513 & 103.48997 & 50.0517 \\
\hline$\frac{1}{2}$ & 18.090 & $\mathrm{BB}$ & 0.638 & 1910.92993 & 46.59590 & 49.9483 \\
\hline
\end{tabular}

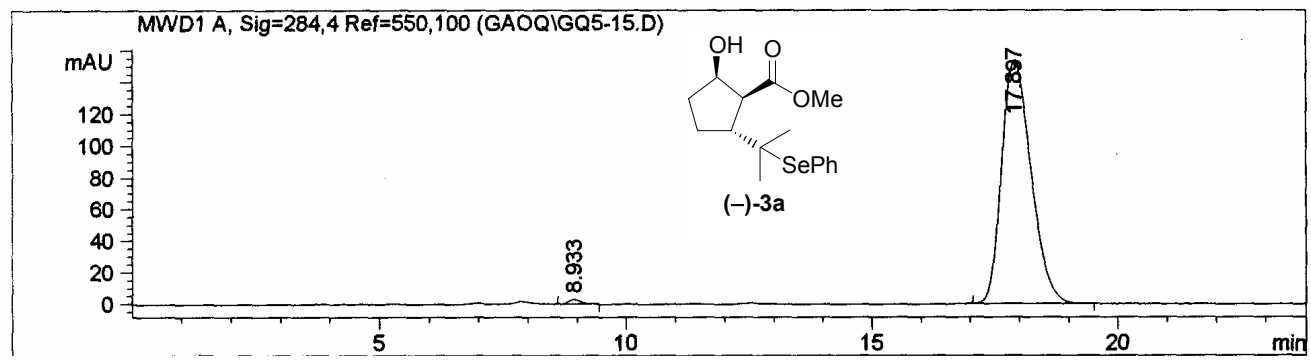

Signal 1: TD1 A, Sig=284,4 Ref $=550,100$

\begin{tabular}{|c|c|c|c|c|c|c|}
\hline $\begin{array}{c}\text { Peak } \\
\text { \# }\end{array}$ & $\begin{array}{c}\text { RetTime } \\
\text { [min] }\end{array}$ & ype & $\begin{array}{l}\mathrm{Wi} \\
{[\mathrm{m}}\end{array}$ & $\begin{array}{c}\text { Area } \\
{\left[\mathrm{mAU}{ }^{*} \mathrm{~s}\right]}\end{array}$ & $\begin{array}{l}\text { Height } \\
\text { [mAU] }\end{array}$ & $\begin{array}{c}\text { Area } \\
\frac{\circ}{6}\end{array}$ \\
\hline & $\begin{array}{r}8 . \\
17 .\end{array}$ & $P$ & & $\begin{array}{r}57.17963 \\
6388.68311\end{array}$ & $\begin{array}{r}3.04770 \\
153.72499\end{array}$ & 99.1129 \\
\hline
\end{tabular}


Column: No: OD OOCE-AH045

Guard column: OD $5 \mathrm{~cm}, 00 \mathrm{CC}-\mathrm{CB} 023$

Solvent: $\mathrm{n}$-hex/ $i-\operatorname{PrOH}=96: 4 ;$ Flow rate: $0.6 \mathrm{ml} / \mathrm{min}$

Sample: gq-5-114

MWD 1: $210 \mathrm{~nm}, 2$ : $209 \mathrm{~nm}$

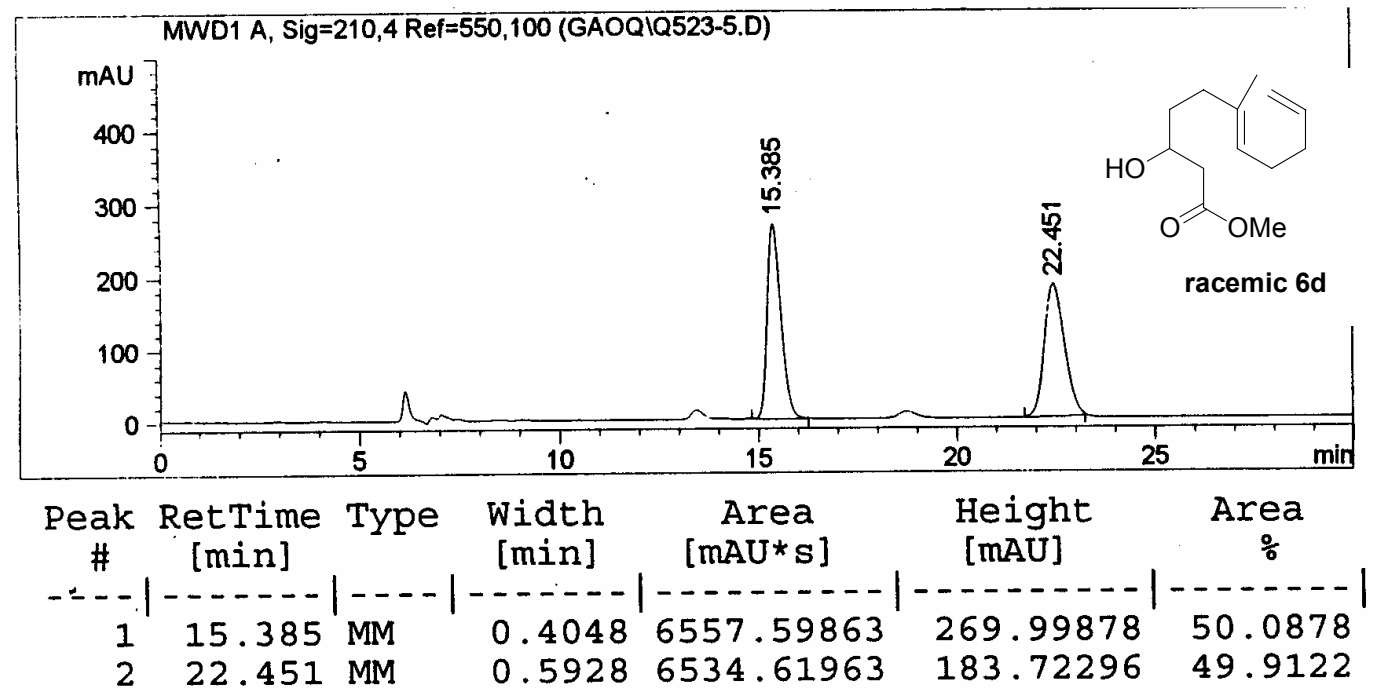

MWD1 A, Sig=2104 Ref $=550,100$ (GAOQIGQ-5-114.D)

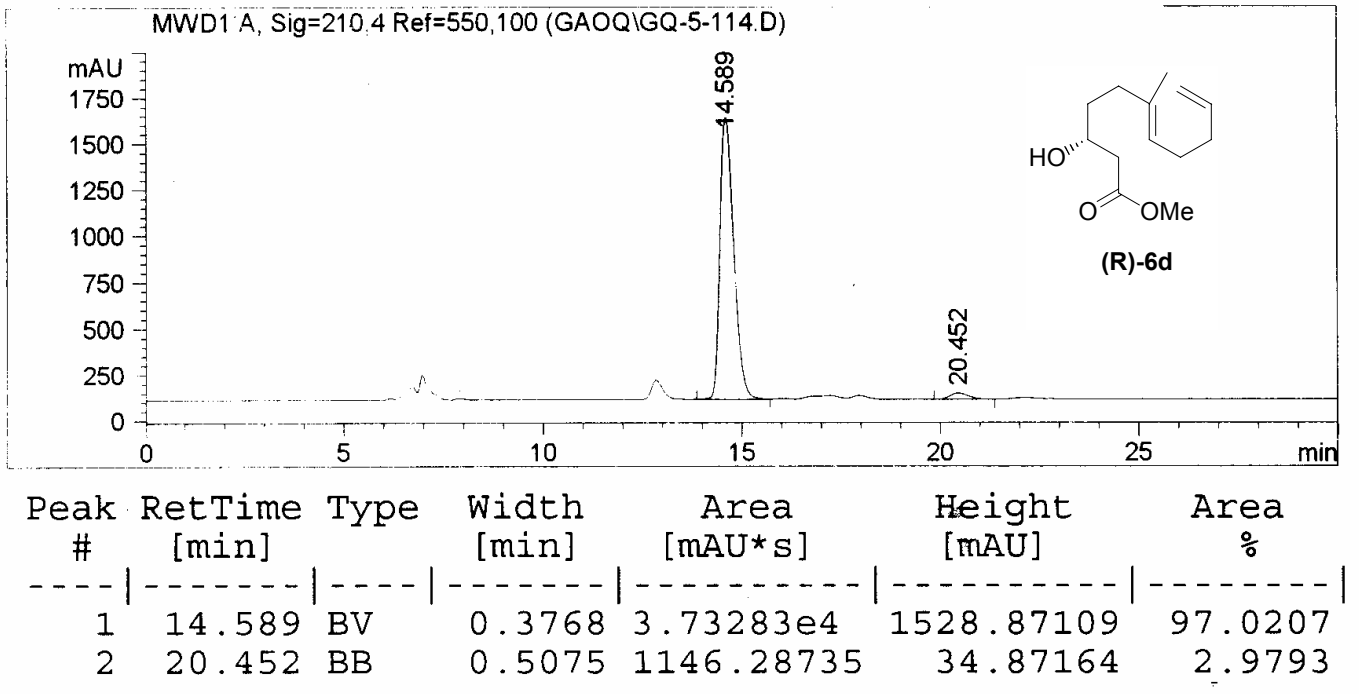


Column: CHIRALCEL OD NO.ODOOCE-Ah045

Guard column: CHIRALCEL ADOOCC-CBO23

Solvent: $\mathrm{n}$-hex/ $i-\mathrm{PrOH}=99: 1 ;$ Flow rate: $0.30 \mathrm{ml} / \mathrm{min}$

Sample: supply-1.rac

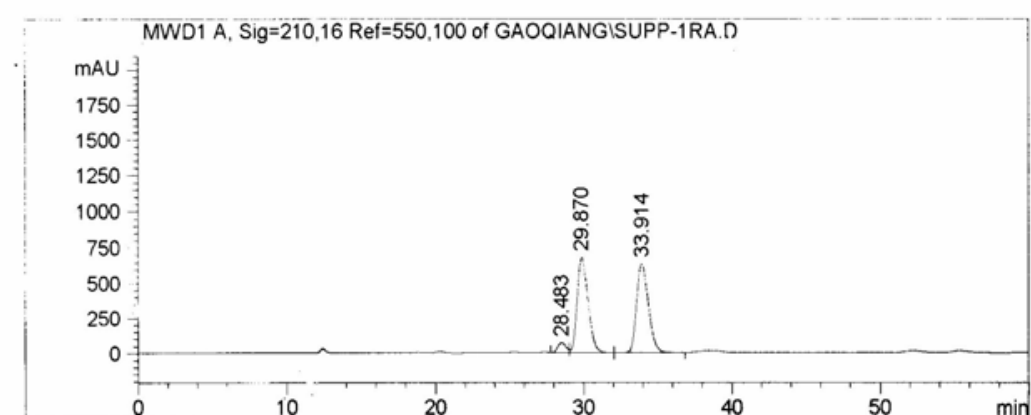

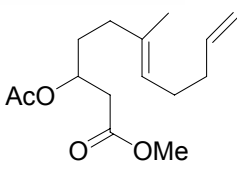

Racemic 7d

10

Type width

$\mathrm{RT}$

[min]

Area

[mAU* $\mathrm{s}$ ]

Height

[mAU]

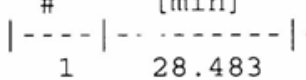

$\begin{array}{ll}1 & 28.483 \\ 2 & 29.870\end{array}$

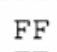

[min]

-...-...-.

$\begin{array}{rr}0.639 & 3010.65991\end{array}$

0.82335600 .65234

73.22249

682.12671

637.15997

Area
$\%$
4.0163
47.4926
48.4910
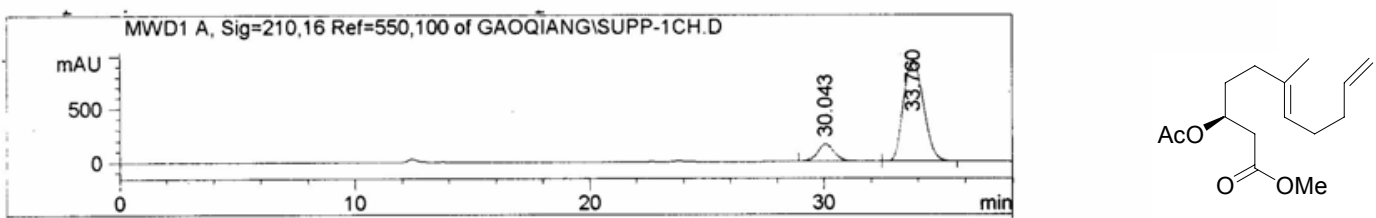

(S)-7d $75 \%$ ee

\begin{tabular}{ccccccc}
$\begin{array}{c}\text { Peak } \\
\text { \# }\end{array}$ & $\begin{array}{c}\text { RT } \\
\text { [min] }\end{array}$ & Type & $\begin{array}{c}\text { Width } \\
\text { [min] }\end{array}$ & $\begin{array}{c}\text { Area } \\
\text { [mAU*s] }\end{array}$ & $\begin{array}{c}\text { Height } \\
\text { [mAU] }\end{array}$ & $\begin{array}{c}\text { Area } \\
\%\end{array}$ \\
\hline 1 & 30.043 & FF & 0.775 & 8362.50000 & 161.93138 & 12.9795 \\
\hline 2 & 33.760 & FF & 0.952 & 56066.06641 & 952.70679 & 87.0205
\end{tabular}


Column : NO: ODOOCE-AHO45

Guard column: OD NO: ODOOCC-CB023

Solvent: $\mathrm{n}$-hex/ i-PrOH $=96: 4$; Flow rate: $0.6 \mathrm{ml} / \mathrm{min}$ Sample:gq-5-4
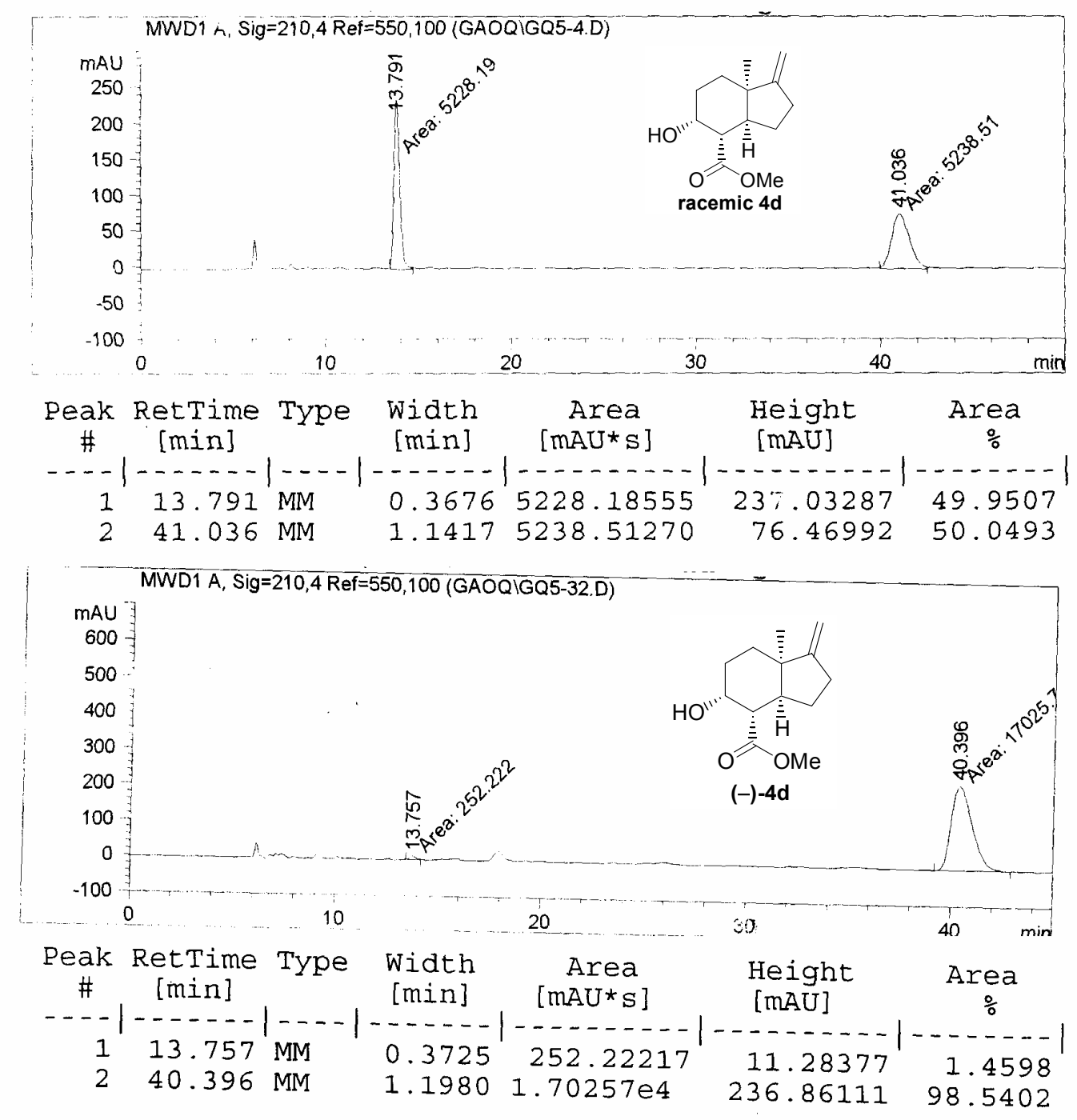
TOlumn: No: OL OOCE-AHO 45

Guard column: OD $5 \mathrm{~cm}$, OOCC-CB023

Solvent: $\mathrm{n}$-hex/ $i-\operatorname{PrOH}=96: 4 ;$ Flow rate $0.6 \mathrm{ml} / \mathrm{min}$

Sample: gq-5-99sm
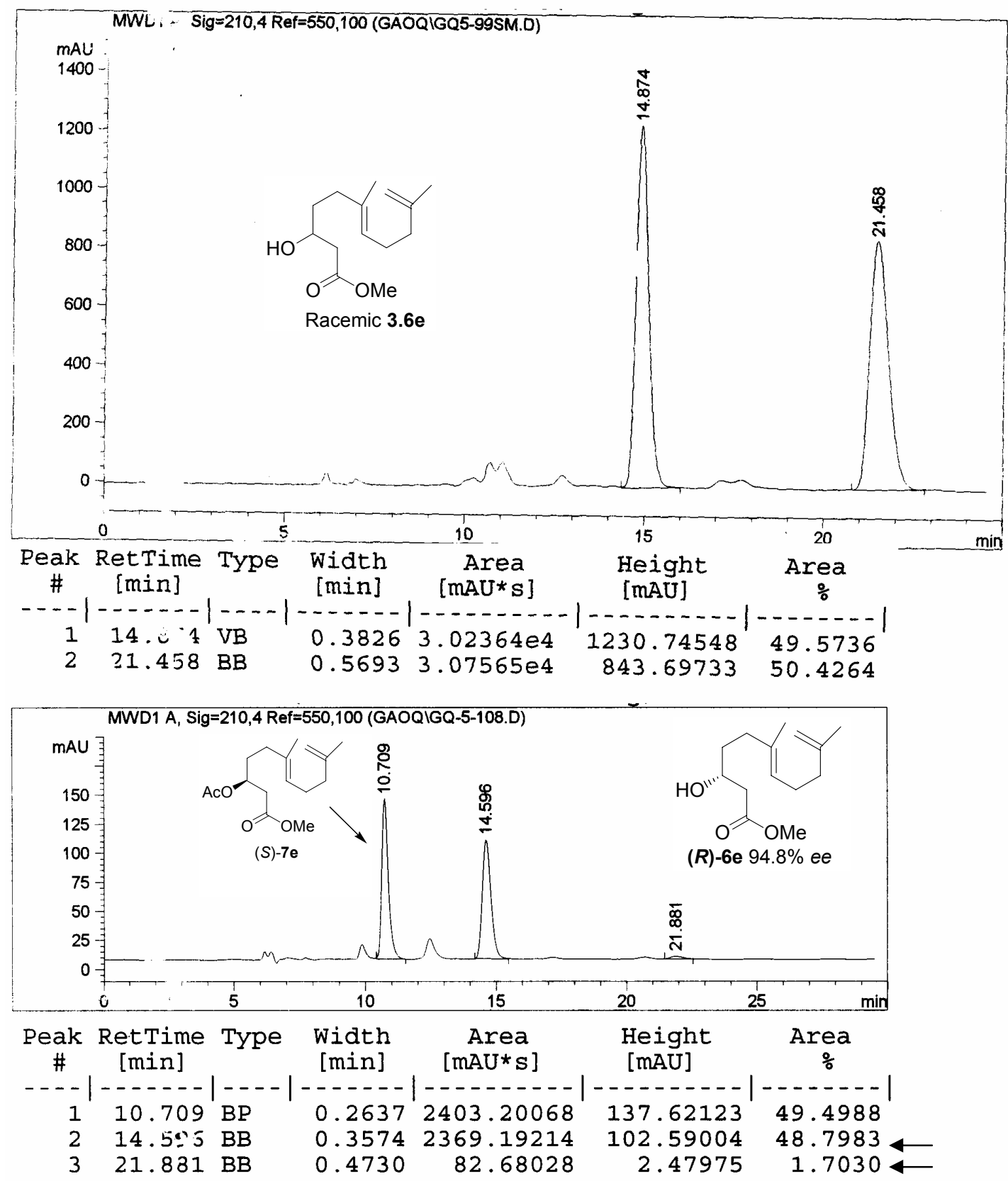
Column: CHIRALCEL OD NO.ODO0CE-Ah045

Guard column: CHIRALCEL ADOOCC-CBO23

Solvent: $\mathrm{n}$-hex/ $\mathrm{i}-\mathrm{PrOH}=99: 1$; Flow rate: $0.30 \mathrm{ml} / \mathrm{min}$

Sample: supply- real racmic
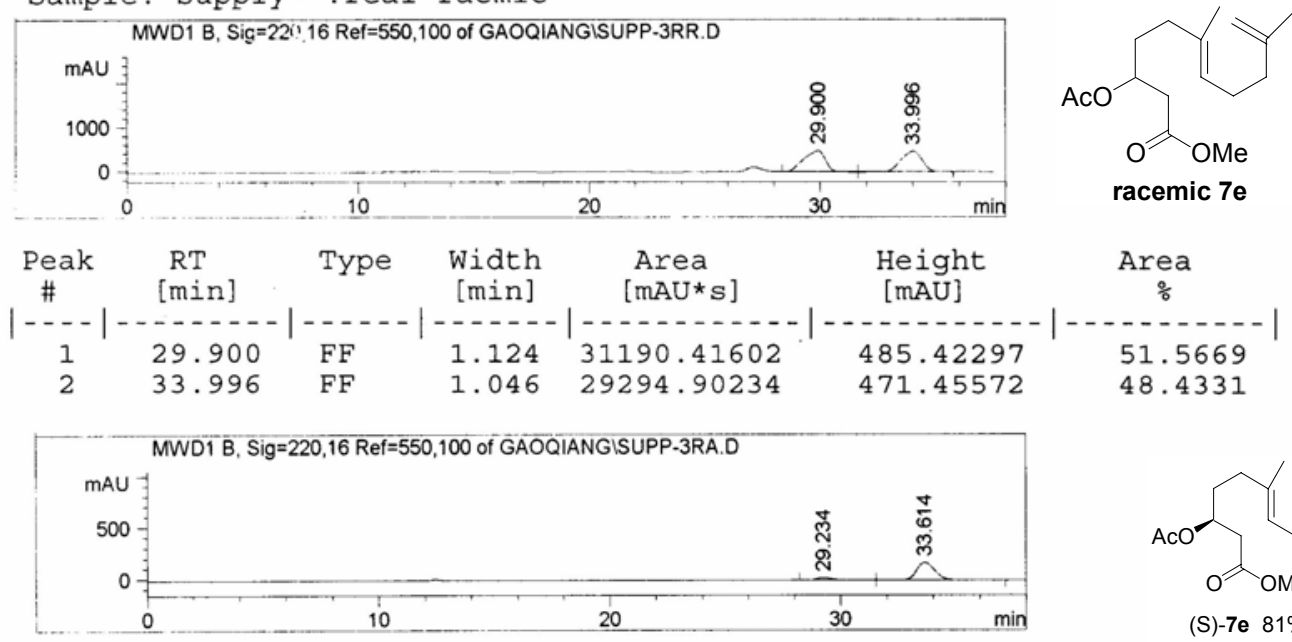

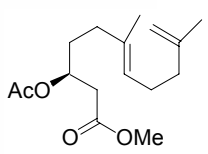

(S)-7e $81 \%$ ee

\begin{tabular}{|c|c|c|c|c|c|c|}
\hline $\begin{array}{l}\text { Peak } \\
\#\end{array}$ & $\begin{array}{l}\mathrm{RT} \\
{[\mathrm{min}]}\end{array}$ & Type & $\begin{array}{l}\text { Width } \\
\text { [min] }\end{array}$ & $\begin{array}{l}\text { Area } \\
{\left[\mathrm{mAU}{ }^{*} \mathrm{~s}\right]}\end{array}$ & $\begin{array}{l}\text { Height } \\
\text { [mAU] }\end{array}$ & $\begin{array}{c}\text { Area } \\
\frac{\%}{6}\end{array}$ \\
\hline & & & & & & \\
\hline $\begin{array}{l}1 \\
2\end{array}$ & $\begin{array}{l}29.234 \\
33.614\end{array}$ & $\begin{array}{l}\text { BF } \\
\text { FB }\end{array}$ & $\begin{array}{l}0.667 \\
0.842\end{array}$ & $\begin{array}{r}964.59265 \\
9026.06348\end{array}$ & $\begin{array}{r}22.09360 \\
168.23712\end{array}$ & $\begin{array}{r}9.6549 \\
90.3451\end{array}$ \\
\hline
\end{tabular}


column: CHIRALCEl AD; No.: AD0OCE-JH192

guard column: chiral Pal AD; No.: AD00CC-CJ023

Solvent: Hexane: i-PrOH $=97 / 3$

flow rate: $0.4 \mathrm{ml} / \mathrm{min}$

Sample name:gq1-11w

MWD1 $: 210,2: 209$,
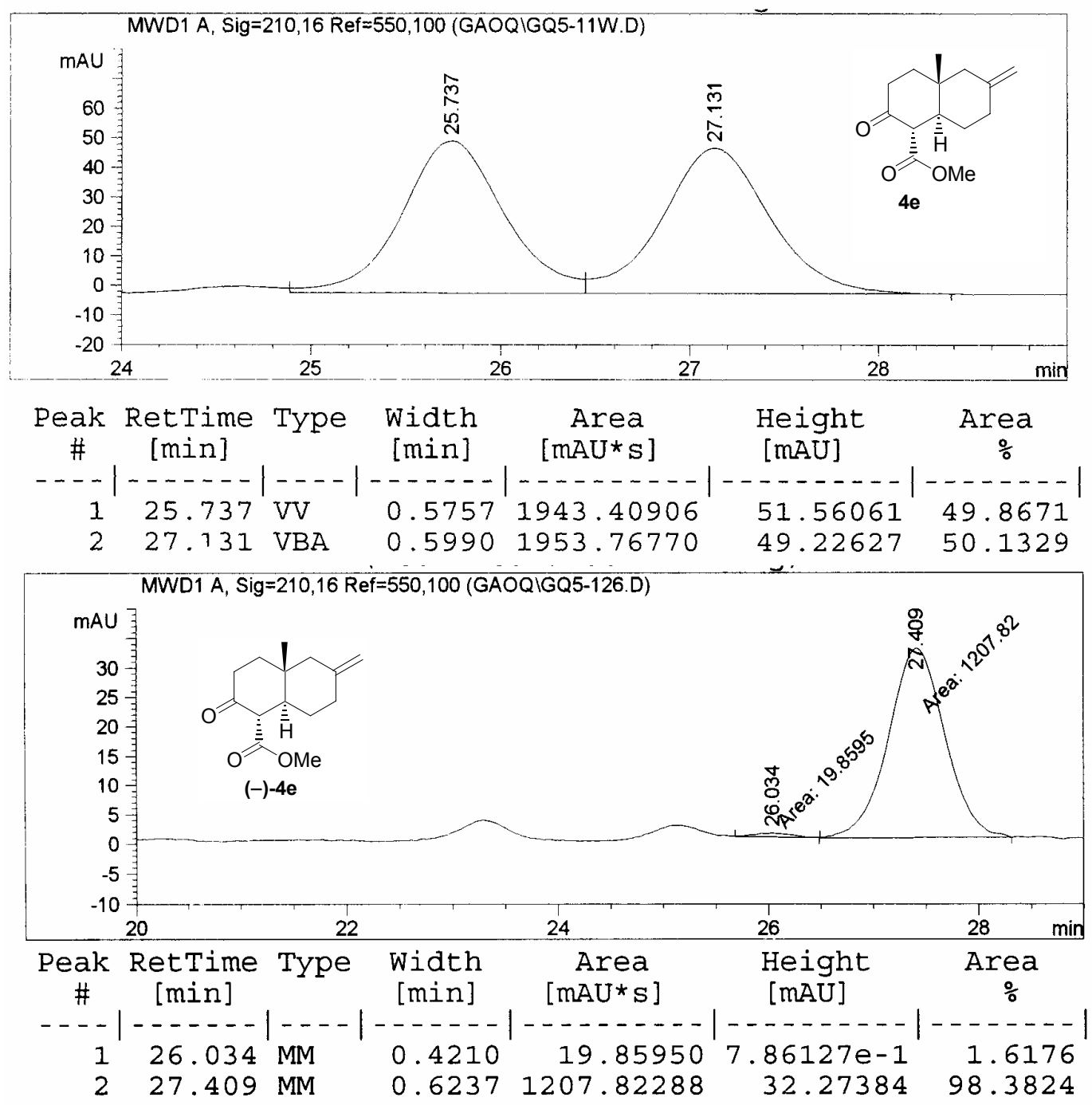